\title{
It wasn't Witchcraft-It was Huntington Disease!
}

\author{
Eribeth Penaranda, MD, Angel Garcia, MD, and Lisa Montgomery, MS
}

Huntington disease (HD) is an autosomal-dominant, incurable, progressive disorder that manifests with chorea and behavioral and cognitive impairment. The disease usually occurs during the fourth or fifth decade of life; however, it may present at any age. Clinical suspicion is confirmed by genetic testing. Death occurs, on average, 15 to 20 years after the onset of symptoms. Here we report about a Hispanic woman and her family who were affected by the disease; this case illustrates the role of cultural values and beliefs in the decision-making process, as well as the importance of the physician's cultural competency in fostering a trusting relationship that may lessen the burden of catastrophic diseases on individuals, families, and society at-large.(J Am Board Fam Med 2011;24:115-116.)

Keywords: Alternative Medicine, Behavior Mechanisms, Doctor-Patient Relationship, Medical Ethics, Neurology

Chief complaint: "no puedo quedarme quieta" ("I can't stay still").

Ms. G. is a 47-year-old Mexican woman who presented to a community clinic on the United States-Mexico border complaining of a 5 -year history of progressively uncontrollable movements; they were initially twitches in her neck and mouth then grew to involve her 4 extremities, and they currently prevent her from independently performing her activities of daily living. She was seeing a curandero, (a Mexican folk healer), who attributed her symptoms to a magic spell that had been placed on all of her family. The curandero treated her with rituals and natural remedies: semilla de uva (grape seed), ojo de gallina (rooster eye), cola de caballo (horse tail), sarsaparilla extract, prune tea, and chamomile tea. Some of her family members have had or currently have similar symptoms (see Figure 1). Physical examination revealed generalized, 2-second choreiform movements, dysarthria, wide-based station gait, and depressed mood. Magnetic reso-

Submitted 22 June 2010; revised 30 June 2010; accepted 6 July 2010.

From the Family and Community Medicine Residency Program, Paul L. Foster School of Medicine, Texas Tech University Health Sciences Center, El Paso, TX (EP, AG, LM).

Funding: none.

Conflict of interest: none declared.

Corresponding author: Eribeth K. Penaranda, MD, Paul L. Foster School of Medicine, Texas Tech University Health Sciences Center, 9849 Kenworthy, El Paso, TX 79924 (Email: eribeth.penaranda@ttuhsc.edu).
Figure 1. Medical genogram of Ms. G.'s family. Affected individuals display signs and symptoms of Huntington Disease according to history.

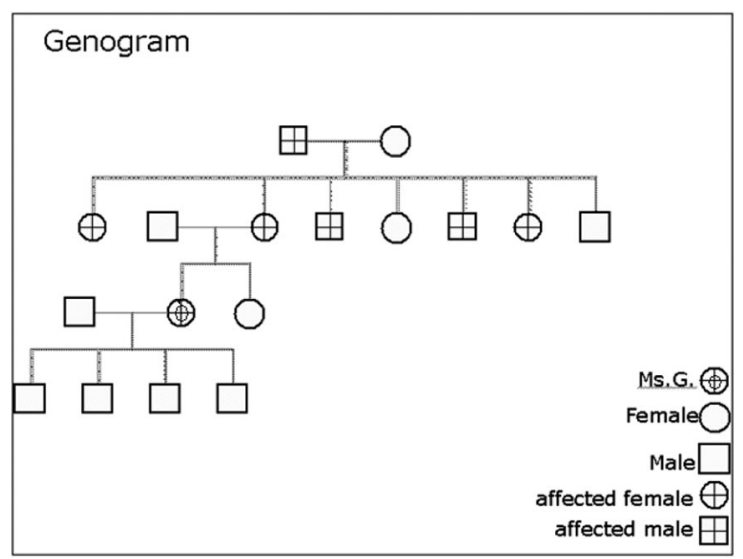

nance imaging of the brain showed diffuse brain atrophy.

Genetic testing showed IT15 allele 1: 46 CAG repeats and IT15 allele 2: 22 CAG repeats, confirming the diagnosis of Huntington disease (HD). Ms. G. was referred to genetic counseling and HD support groups. Her offspring refused genetic testing.

\section{Discussion}

HD is an autosomal-dominant disorder that occurs in 4 to 5 of every 1 million people worldwide, and it is more common in Western countries. It is caused by an abnormal expansion of the trinucleotide CAG tandem repeat in the IT15 gene in the chromosome 4p. 
The polyglutamine repeats (CAG) accumulate from one generation to the next, causing intergenerational anticipation. Onset usually occurs by the fourth to fifth decade of life; however, it may present at any age. HD manifests as gradually worsening chorea and behavioral and cognitive impairment, such as depression and psychosis. Other symptoms and signs include impaired fine motor coordination, weight loss, dysarthria and dysphagia abnormal eye movements, impairment of word fluency and lack of insight into the cognitive problem, rigidity, and akinesia in advanced stages. ${ }^{1}$

Diagnosis is made through the clinical features along with family history, and is confirmed by genetic testing. Neuroimaging may show caudate atrophy, which can explain the cognitive impairment. Because there are not medications to slow the progression of HD, therapy remains supportive. Medications such as tetrabenazine reduce severe choreal psychosis and depression are managed accordingly. Death occurs between 15 to 20 years after the onset of symptoms, mainly as a consequence of complications of immobility. The suicide rate is as high as $7 \%$. Genetic testing is available for at-risk individuals to determine carrier states. Prenatal testing through chorionic sampling is available. Testing at a multidisciplinary clinic before symptoms present can provide individuals with the necessary support. ${ }^{1,2}$

\section{HD in Latin America}

The study of the world's largest HD concentration in Venezuela through the "Venezuela HD Project" led to the final isolation of the HD gene in $1993 .{ }^{3}$ Distinctive disease characteristics among Hispanics include increased incidence of infantile cases, shorter disease duration, lower suicide rates, and lower likelihood of pregnancy termination if prenatal testing is positive when compared with other races. $^{4,5}$

\section{Cultural Competencies}

Traditional values within the Hispanic culture include the belief that diseases are caused by possession by evil spirits, for which relief is sought via curanderos, frequently in conjunction with allopathic medicine. Curanderos are considered to be gifted people knowledgeable about alternative therapies such as herbs, rituals, spiritual healing, prayer, and massage. ${ }^{6}$

HD is such a debilitating disease that affected or at-risk individuals face many ethical dilemmas, including their reproductive choices, such as becoming pregnant, undergoing prenatal testing, and terminating a pregnancy. Extensive counseling by genetic units that specialize in the disease and interdisciplinary support is highly recommended. This case illustrates the importance of cultural competency in fostering a trusting relationship that may lessen the burden of catastrophic diseases on individuals, families, and society at-large.

\section{References}

1. Walker FO. Huntington's disease. Semin Neurol 2007;27:143-50.

2. Ho LW, Carmichael J, Swartz J, Wyttenbach A, Rankin J, Rubinsztein DC. The molecular biology of Huntington's disease. Psychol Med 2001;31:3-14.

3. Hereditary Disease Foundation. The Venezuela Huntington Disease Project. 2008. Available at: http:// www.hdfoundation.org/html/venezuela_huntington. php. Accessed June 15, 2010.

4. Drake H, Reid T, Marteau T. Attitudes towards termination for fetal abnormality: comparisons in three European countries. Clin Genet 1996;49:134-40.

5. Alonso ME, Ochoa A, Boll MC, et al. Clinical and genetic characteristics of Mexican Huntington's disease patients. Mov Disord 2009;24:2012-5.

6. Tafur MM, Crowe TK, Torres E. A review of curanderismo and healing practices among Mexicans and Mexican Americans. Occup Ther Int 2009;16:82-8. 